\title{
Affective consequences and subsequent effects on morphine self-administration of $\mathrm{d}$-ala ${ }^{2}$-methionine enkephalin
}

\author{
JUNE M. STAPLETON, MARCIA D. LIND, VICKI J. MERRIMAN, \\ MICHAEL A. BOZARTH, and LARRY D. REID \\ Rensselaer Polytechnic Institute, Troy, New York 12181
}

\begin{abstract}
Albino rats implanted with intracerebroventricular (ICV) cannulae were used to investigate the effects of ICV administration of $d$-ala ${ }^{2}$-methionine enkephalin. Potentially positive affective consequences were assessed by observing rats' movements in an alley, one compartment of which had previously been paired with drug administration. Like morphine $(10 \mathrm{mg} / \mathrm{kg}$, intraperitoneally-IP), this enkephalin analogue $(10 \mu \mathrm{g}$, ICV) produced a tendency for rats to move toward the place where they had previously experienced the drug's effects. In another experiment, the same dose of $d-$ ala $^{2}$-methionine enkephalin was not sufficient to produce a conditioned taste aversion, as did the $10-\mathrm{mg} / \mathrm{kg}$ IP dose of morphine. Rats with a prior history of administration of either $\mathrm{d}-\mathrm{ala}^{2}$-methionine enkephalin or systemic morphine subsequently consumed significantly more sweetened morphine solution than control animals in a voluntary oral consumption situation with tap water also available. Collectively, these results suggest that enkephalin administration may produce a positive affective state without aversive components and potentiate voluntary consumption of morphine.
\end{abstract}

Morphine-like opioids have a variety of effects including, of course, analgesia and the capacity to reinforce the act of taking them. Reinforcement for taking opioids involves the elicitation of a positive affective state (Adams, Lorenz, \& Mitchell, 1972; Esposito \& Kornetsky, 1978; McAuliffe \& Gordon, 1974), although the extent of the influence of these positive elements is controversial (Criswell \& Levitt, 1975; Wikler, 1974). Regardless of the details of the reinforcement process following opioid assimilation, it is apparent that opioid reinforcement seems to be more potent after considerable assimilation of opioids. A question addressed by these experiments is whether an analogue of enkephalin, an endogenous opioid, will also elicit positive affect, as indexed by rats' movements to the place where they had previously experienced the effects of the enkephalin, and whether previous exposure to the enkephalin will potentiate subsequent intake of morphine.

There are other implications that can be derived from studies of the effects of endorphins on reinforcement and motivation. Any central nervous system neurotransmitter or modulator whose effective concentrations might deviate from a norm, while that deviation is not severely debilitating or lethal, is a candidate for being a transmitter related to a class of mental illness. Consequently, there are potentially

Supported by Grant DA 02044 from the National Institute on Drug Abuse. M. Bozarth is now with the Department of Psychology, Concordia University, Quebec, Canada. The assistance of Constance L. Coogle is gratefully acknowledged. as many hypotheses of affective disorders as there are combinations of transmitters and classifications of disorders, and there are endorphin hypotheses of affective disorders. Although there are theoretical reasons and some empirical support for an endorphin hypothesis of affective disorders (Bloom, Segal, Ling, \& Guillemin, 1976; Jacquet \& Marks, 1976; Watson, Berger, Akil, Mills, \& Barchas, 1978), there is little evidence suggesting that changes in endorphins can in turn lead to affective or motivational changes (Grevert \& Goldstein, 1978). Belluzzi and Stein (1977) have shown that there is a relationship between endorphins and intracranial selfstimulation, and that endorphins will be selfadministered. Some of the basic observations of Belluzzi and Stein (1977), however, have not been observed by others (Van der Kooy, LePiane, \& Phillips, 1977). The studies reported in this paper do indicate that an endorphin analogue, in relatively large doses, may produce a positive affective state and that an effect endures beyond endorphin administration. These observations, therefore, are not incompatible with speculations that link potential endorphin function or malfunction to affective and motivational processes.

\section{EXPERIMENT 1}

This was our first assessment of the affective consequences of the administration of $\mathrm{d}$-ala ${ }^{2}$-methionine enkephalin, a long-lasting enkephalin analogue (Walker, Sandman, Berntson, McGivern, Coy, \& Kastin, 
1977). The method used to index the putative affective state due to enkephalin administration was the method used by Rossi and Reid (1976) to assess morphine's consequences.

\section{Method}

Subjects. The subjects were 40 experimentally naive, adult, male, albino (Taconic Farms) rats weighing 275 to $475 \mathrm{~g}$ at the start of the procedures. Throughout the experiment, each was housed individually with food and water always available.

Twenty-four rats were each fixed, using standard stereotaxic procedures, with a chronically indwelling, stainless steel guide cannula. The 23-ga guide was placed so that infusions of fluids would be in the lateral ventricle. The stereotaxic coordinates for the tip of the guide were $.9 \mathrm{~mm}$ anterior to bregma, $1.5 \mathrm{~mm}$ lateral to the midline, and $3.5 \mathrm{~mm}$ below the top of the skull with bregma and lambda in the same horizontal plane, perpendicular to the cannula's shaft. Sodium pentobarbital anesthesia $(45 \mathrm{mg} / \mathrm{kg})$ was used, with atropine sulfate as an adjunct. At the time of surgery, a dummy cannula of 30 -ga stainless steel wire, $.5 \mathrm{~mm}$ longer than the guide, was inserted and left in place except during infusions.

After completion of all behavioral testing, the rats were sacrificed using an overdose of sodium pentobarbital, and intracardially perfused with physiological saline followed by a $10 \%$ Formalin solution. For histological verification of cannula placements, $90-\mu$ frozen sections of brain were treated as photographic negatives to produce an enlarged $(\times 10)$ photograph of the section.

Apparatus. A wooden alley $(98 \times 23 \times 29 \mathrm{~cm})$ was used to assess preference for the place of drug experience. The alley was divided into three compartments by two guillotine doors. The middle compartment $(8 \times 23 \times 29 \mathrm{~cm})$ was gray and had a solid wooden floor. A $45 \times 23 \times 29 \mathrm{~cm}$ chamber was on each side of the middle compartment. One side chamber had alternating black and white vertical stripes on the walls and a $1.3 \times 1.3 \mathrm{~cm}$ wiremesh floor. The other side chamber had alternating black and white horizontal stripes and a $1.3 \times 2.5 \mathrm{~cm}$ wire mesh floor. The alley was the same one used by Rossi and Reid (1976), except that the walls were striped rather than being black on one side and white on the other side.

Procedure. Prior to the procedures of fixing the cannulae, each subject was randomly assigned to one of five groups $(n=8$ / group). On certain days of the procedures, each member of a group received one of the following drug treatments prior to being placed in the apparatus: (a) $10 \mu \mathrm{g}$ of d-ala ${ }^{2}$-methionine enkephalin (from Peninsula Laboratories, lot No. 801013) administered intracerebroventricularly (ICV), (b) physiological saline, ICV, (c) $10 \mu \mathrm{g}$ of morphine sulfate, ICV, (d) $10 \mathrm{mg}$ of morphine sulfate $/ \mathrm{kg}$ of body weight given intraperitoneally (IP), or (e) physiological saline, IP. All ICV infusions were $5 \mu \mathrm{l}$ and IP injections were $1 \mathrm{ml} / \mathrm{kg}$. Physiological saline was the carrier of the drugs. ICV infusions were done with a 10- $\mu$ l syringe (Hamilton No. 701) equipped with a 30-ga injection cannula. The injection cannula extended $1.0 \mathrm{~mm}$ beyond the tip of the rat's guide cannula.

Following at least 5 days' recovery from surgery, each rat was placed in the alley for $30 \mathrm{~min}$ on each of 2 consecutive days, allowing it some opportunity to become familiar with the apparatus. A rat was placed in the middle compartment, and after $30 \mathrm{sec}$ the doors were opened, giving it access to the entire alley.

On each of the next 3 days, each rat received an injection or infusion of its assigned drug immediately before being placed in one of the side compartments for conditioning. In each group, one-half of the rats received their putative conditioning in the side with horizontal stripes and one-half received theirs in the side with vertical stripes. Each rat remained in its compartment for $30 \mathrm{~min}$ and then was returned to its home cage. On the next day, a testtrial day, a subject received neither an infusion nor an injection. It was placed in the middle compartment for $30 \mathrm{sec}$ followed by 15-min access to the entire alley. This 4-day regimen (three daily sessions of putative conditioning and 1 day of testing) occurred four times over 16 consecutive days. Thus, four tests were given of a subject's tendency to be in the side in which it had experienced the drug state.

The observations during a test session were done by individuals who were blind to the rats' previous experience. For each side chamber, they recorded the time a rat spent with at least three paws in that chamber. Consequently, there are three possible scores: (a) a score for the time in the putative conditioning chamber, (b) a score for the time in the other side chamber, and (c) a score for the time remaining, which includes time in the middle chamber and the time when a rat did not have three paws in either side chamber. These scores can also be transformed into ratios or difference scores. We have found, however, that many ways of tabulating these kinds of data yield similar summaries of the rats' performances. Consequently, we used the simplest tabulation, the time spent in the side of putative conditioning, in further analyses.

The experimental design conforms to a model for a 5 by 2 by 4 factorial analysis of variance (ANOVA), having repeated measures on the last factor, with a factor associated with the five types of drug treatments, the two sides of the alley (horizontal or vertical stripes), and the four tests. There were eight scores per cell, with the exceptions mentioned below, and each score was the number of seconds a rat stayed in the side of putative conditioning. One of the rats scheduled to be treated with enkephalin had a needle broken off in its guide cannula during the experiment. Two systemic placebo rats mistakenly received conditioning in the wrong side of the apparatus. Consequently, these subjects' scores are not included in any analyses, leaving a total of 37 rats.

\section{Results}

The repeated-measures ANOVA on the scores yielded an $F(4,27)=3.69, p<.02$, for the factor associated with the various drug treatments. No other main effect or interaction was a reliable source of variance (Fs $<1.4$, ps $>.25$ ). The mean time each group spent on the side of putative conditioning across all four test sessions is presented in Figure 1. Using $t$ tests for comparing independent groups and using for each subject the mean time spent on the side of putative conditioning as the score, further comparisons of the groups' performances were made. The scores of the group receiving morphine IP were reliably larger than the group receiving saline IP $[t(14)=2.69, p<.02]$, thereby confirming the results from very similar experiments by Reid, Lind, Bozarth, Merriman, and Stapleton (1978) and Rossi and Reid (1976). The group receiving d-ala ${ }^{2}$-methionine enkephalin spent reliably more time in the side of conditioning than the group receiving saline ICV $[t(13)=3.14, p<.01]$. The scores of the group receiving morphine ICV were not reliably different from the control groups' scores, nor were the two control groups' scores reliably different from each other. Post hoc comparisons using Duncan's multiple range test produced values leading to the same conclusion. In summary, both systemic morphine and $\mathrm{d}$-ala ${ }^{2}$-methionine enkephalin produced a tendency for the rats to spend more time in the place where they had experienced their respective drugs' effects than did groups receiving a placebo. 


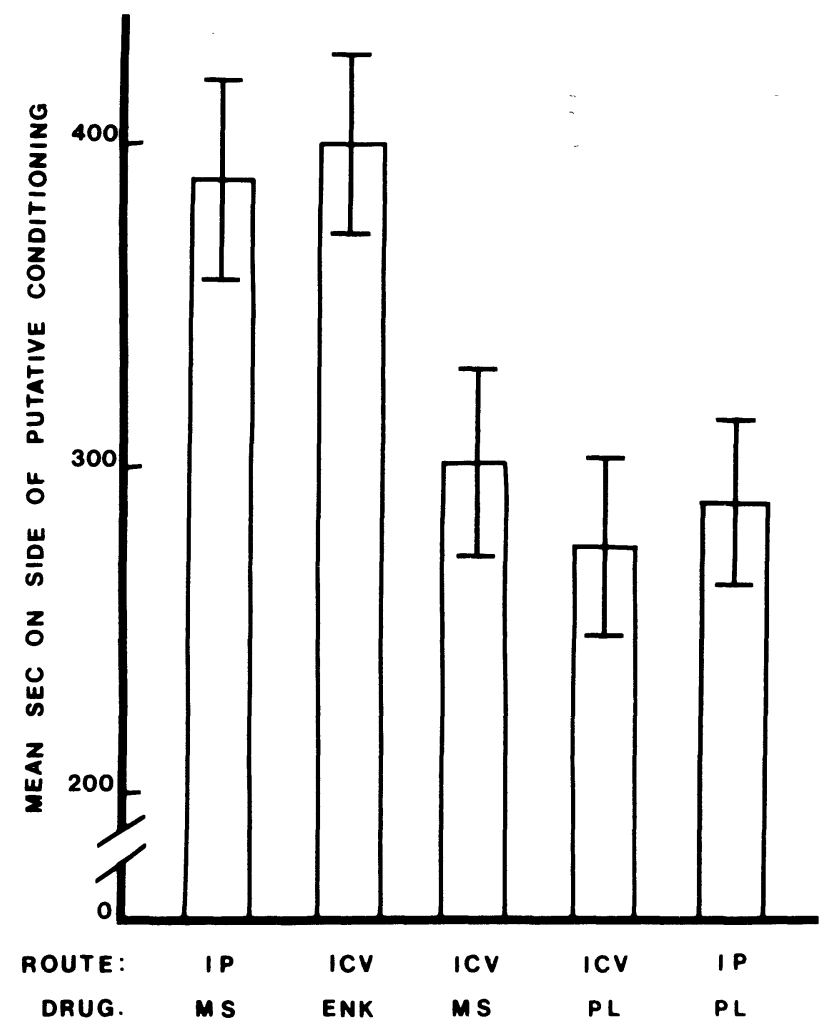

Figure 1. Mean seconds ( \pm SEM) in side of putative conditioning for each drug condition across subjects and trials. MS = morphine sulfate, ENK $=$ d-ala ${ }^{2}$-methionine enkephalin, $P L=$ placebo, physiological saline. $I P=$ intraperitoneally, ICV = intracerebroventricularly.

Histological examination indicated that nearly all cannula tips penetrated into the ventricles. When analyses were done excluding the results from the few subjects for which it was doubtful whether the infusions were in the ventricle, the same general results were achieved. The infusions or the cannula tips frequently caused slight damage to the caudate nucleus, lateral septal nucleus, or the area of the hippocampal commissure. In nearly all relevant sections, there was some necrotic tissue near the place where the infusion must have entered. Since the lesions were small and since the ICV-placebo group performed very similarly to the IP-placebo group, it is concluded that the lesions produced by the ICV procedure were not a factor in determining the outcome of the experiment.

Immediately after the initial doses of enkephalin, the rats showed head shaking and a stereotyped tremor of the forepaws, effects similar to those previously reported (Bloom et al., 1976). The subjects getting ICV morphine showed few signs of sedation, whereas the rats getting morphine IP showed both sedation and occasionally catatonia. Although no formal measures were taken, all of the observed effects following opioid administration waned with repeated dosing, i.e., tolerance was seen.

\section{Discussion}

Since rats tend to move to the place where they had previously experienced the effects of systemically administered morphine, it would be expected that they would also move to the place where they had experienced ICV-administered morphine. The doses of morphine were, however, somewhat arbitrarily selected. Our informal observations led to the conclu-

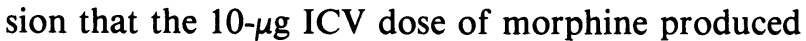
only slight effects, particularly when compared to the $10-\mathrm{mg} / \mathrm{kg}$, IP dose. As a further test of the hypothesis that the ICV-administered morphine was a small dose, the following procedures were done with a separate group of 16 rats.

Thirty minutes after an ICV infusion or IP injection of various doses of morphine or placebo, the rat's motor activity was measured in two ways: (a) number of crossings performed during $90 \mathrm{sec}$ in a standard shuttlebox $(23 \times 23 \times 50 \mathrm{~cm})$, and (b) number of centimeters traveled forward in $90 \mathrm{sec}$ in a $\mathrm{C}$-shaped runway. The scores for $10 \mu \mathrm{g}$ of morphine ICV were not significantly different from placebo scores on either measure $[\operatorname{ts}(6)=1.55,1.00$, respectively, ps $>.15$ ], while $10 \mathrm{mg} / \mathrm{kg}$ of morphine IP produced a significant decrease in activity on both measures $[\operatorname{ts}(7)=4.98,3.28$, respectively, ps $<.05]$. For example, the mean number of shuttles in $90 \mathrm{sec}$ under $10 \mathrm{mg} / \mathrm{kg}$ morphine IP was only $1.29 \mathrm{com}$ pared to 3.42 and 3.08 for IP placebo and ICV placebo, respectively. Ten micrograms of morphine ICV produced shuttling similar to placebo, mean = 3.71. Morphine ICV in larger doses, 20 and $40 \mu \mathrm{g}$, produced mean shuttle scores of 1.58 and 1.00 , respectively, suggesting that effects equivalent to $10 \mathrm{mg} / \mathrm{kg}$ IP would require between 20 and $40 \mu \mathrm{g}$ ICV.

To get some idea of the propensity for the rats to learn the discrimination called for in this experiment, five other rats were trained (with no drug treatment) to leave the startbox and go to one side of the alley for food. Without habituation to the chamber, they were given 12 trials/day across 8 consecutive days to go to food when deprived of food for $24 \mathrm{~h}$. Cues, such as the way the rat was placed in the startbox and the orientation of the alley in the room were controlled for by varying these parameters unsystematically. By the 8th daily session, the rats were moving to the side of the alley were they had previously received food on $80 \%$ of the trials. This result demonstrates that the rats can readily distinguish the two sides of the alley and that rats, without habituation to the apparatus, engage in motivationally relevant behavior rather quickly.

From the experiment itself and the accompanying tests of motor activity, it seems that the dose of $10 \mu \mathrm{g} \mathrm{ICV}$ of morphine is too small to produce measurable effects. The tests were, however, sensi- 
tive to the effects of $10-\mathrm{mg} / \mathrm{kg}$ injections of morphine. Since the test reputedly measuring positive affect also allowed the conclusion that enkephalin produced an effect, it seems reasonable to conclude that systemic morphine and ICV-administered d-ala ${ }^{2}$ methionine enkephalin, although producing different observable behaviors, may both produce a positive affective state, as indexed by the procedures used by Rossi and Reid (1976).

\section{EXPERIMENT 2}

The purpose of these procedures was to see whether a history of $\mathrm{d}$-ala ${ }^{2}$-methionine enkephalin administration would potentiate voluntary consumption of sweetened morphine solutions.

\section{Method}

The subjects of this procedure were the subjects of Experiment 1 ( $n=37)$. Water bottles, equipped with ballpoint sipping tubes and attached to the rats' home cages, were used to measure fluid consumption.

Six days after the last drug administration of Experiment 1, all rats were given an opportunity to consume sweetened morphine solution in a two-bottle choice situation, the other bottle containing tap water. The morphine solution was $.5 \mathrm{mg}$ morphine sulfate for every milliliter of solution with $10 \%$ sucrose $(w / v)$ in tap water, a solution similar to that used by Khavari and Risner (1973). The bottles were weighed, fresh solutions provided, and the positions of the two bottles alternated every $48 \mathrm{~h}$.

\section{Results and Discussion}

The results are shown in Figure 2. The scores from which Figure 2 was derived were submitted to a 5 by 6 ANOVA having repeated measures with factors of the five groups having different histories of drug administration and the six repeated measurements of morphine consumption. The results of the ANOVA indicated a reliable effect for the factor of drug history $[F(4,32)=3.95, p<.02]$ and for the factor of trials $[F(5,160)=4.64, p<.001]$. The interaction of Group by Trials was not a statistically significant source of variance $(F<1)$. Mean consumption across all 12 days was greater for the enkephalin group than for the ICV placebo group [t(13) $=2.79, \mathrm{p}<.03$ ] Overall, the systemic morphine group consumed more sweetened morphine solution than the systemic placebo group $[\mathrm{t}(14)=2.42, \mathrm{p}<.04]$. The ICV morphine group was not significantly different from the ICV placebo group, nor were the two placebo groups significantly different with respect to their consumption.

Measures of oral consumption of sweetened morphine solutions are potentially useful, but they have an inherent confounding factor, namely palatability. Since sweetener was used to mask the bitter taste of morphine, any effect shown with these measures could conceivably index a change in reactivity to sucrose rather than morphine. The work of Khavari

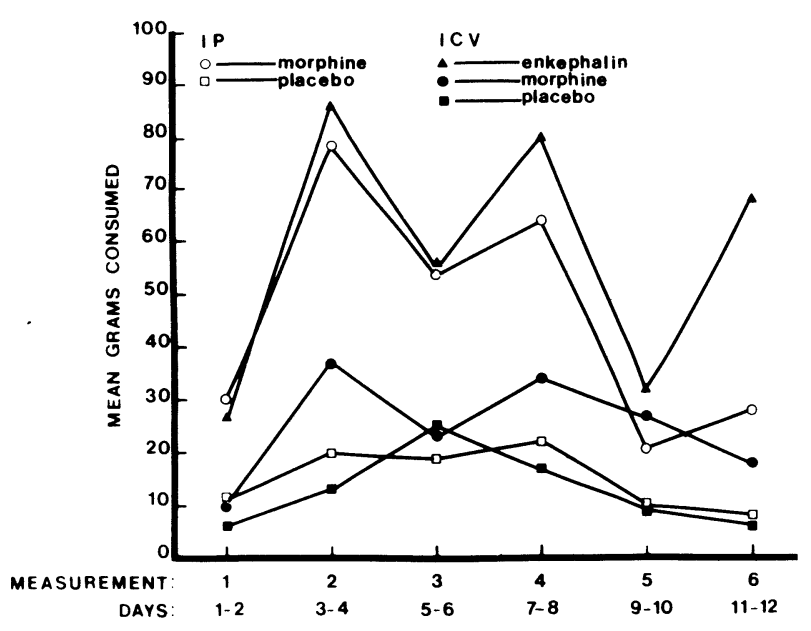

Figure 2. Mean grams of sweetened morphine solution taken each $48 \mathrm{~h}$ of availability. Each group had a different history of drug administration.

and Risner (1973), however, indicates that rats previously addicted to morphine will choose to drink a $10 \%$ sucrose solution with $.5 \mathrm{mg} / \mathrm{ml}$ morphine in preference to the same sucrose solution without morphine. Unpublished results from our laboratory indicate that 12 to 15 doses of $10 \mathrm{mg} / \mathrm{kg} /$ day of morphine is sufficient to potentiate initial consumption of sweetened morphine solutions, a finding confirmed by this study and Gorman, DeObaldia, Scott, and Reid (1978). We have also found that massive dosing with morphine, for example, two doses per day for 8 days of ascending doses ending with $200 \mathrm{mg} / \mathrm{kg} /$ day, may or may not potentiate subsequent consumption. Consequently, there are regimens of dosing with morphine that reliably potentiate initial consumption of sweetened morphine solution. Apparently, there are also dosing regimens of enkephalin that will potentiate intake of morphine solutions.

The conclusion of the present study that exposure to enkephalin or morphine may potentiate selfadministration of morphine needs confirmation using oral self-administration of etonitazene, a potent, tasteless opioid, or intravenous self-administration of morphine. Each of these ways of measuring propensity to take opioids is potentially confounded by a different extraneous variable. Concordant results on all three kinds of measures of propensity to take morphine would provide strong support for the conclusion that enkephalin potentiates opioid self-administration. We have no way of knowing, at this time, whether the level of opioid activity produced by the infusions is similar to what might be produced within the natural history of an individual. It is, however, of interest to point out that the effects measured here occurred some days after enkephalin administration and, therefore, potentially represent an enduring effect of high levels of endorphin activity. 


\section{EXPERIMENT 3}

Experiment 3, which was conducted concurrently with Experiment 1, was designed to further assess the affective state produced by the agents and doses used in Experiment 1. The experiment used the now classical taste aversion paradigm (Garcia, Hankins, \& Rusiniak, 1974; Riley \& Baril, 1976). It has been shown that morphine, when used as the unconditioned stimulus, can sustain a poison aversion (LeBlanc \& Cappell, 1974; Parker, Failor, \& Weidman, 1973), but the resultant poison aversion is less intense than those sustained by unequivocally toxic agents (Gorman et al., 1978; Riley, Jacobs, \& Lolordo, 1978). The taste aversion paradigm is theoretically sensitive to the ability of an agent to produce nausea and, combined with results indexing the possibility for an agent to elicit positive effect, can be used to determine if an agent produces negative effects or mixed affective events.

\section{Method}

Subjects and Apparatus. The subjects were 35 experimentally naive, adult, male albino rats (Taconic Farms, Inc.) weighing 225 to $425 \mathrm{~g}$ at the start of procedures. The rats were randomly assigned to one of five drug groups to receive the same drugs and doses as the five groups of Experiment 1 . Those subjects that were to eventually receive infusions ICV were, of course, fitted with cannulae using the same procedure described in Experiment 1.

The subjects were individually housed with free access to food and access to water as specified. Water bottles, equipped with ballpoint sipping tubes and attached to the rats' home cages, were weighed to an accuracy of $1 \mathrm{~g}$ to determine the amount of fluid consumed.

Procedure. Five days after surgery, the rats were deprived of water for $24 \mathrm{~h}$. Water was then presented for $15 \mathrm{~min} /$ day for
6 days, so that the animals could learn to consume a daily ration of water in the time allotted. On the 7 th day, the amount of water consumed was recorded. On Day 8 and every 4th day thereafter, a $.1 \%$ solution of saccharin was presented for $15 \mathrm{~min}$ instead of water. Immediately after removal of the saccharin solution, each rat received an injection or infusion of its assigned drug. Saccharin solutions were presented four times and followed by injections/infusions each time except the last. On intervening days, water was made available for $15 \mathrm{~min} /$ day. This procedure yielded one measure of consumption of saccharin solution prior to any drug administration and three measures subsequent to the pairing of saccharin solutions with administration of morphine, enkephalin, or placebo. Following the saccharin presentations, a final day's consumption of water was recorded. Three animals of the ICV morphine and two animals of the enkephalin groups failed to complete the procedures due to illness or cannula blockage and their scores were deleted from all data analyses, making the $\mathbf{N}=30$.

\section{Results and Discussion}

Mean fluid consumptions are shown in Figure 3. There were no significant differences among the five drug groups in initial or final water consumption as indexed by one-way ANOVAs $[\mathrm{Fs}(4,25)=.69,1.45$, respectively, ps $>.60$ ], indicating that the groups were similar with respect to fluid consumption except when under the experimental procedures. The scores associated with saccharin consumption were submitted to a 5 by 4 ANOVA having repeated measures on the last factor, with factors associated with five drug groups and four trials. It yielded a reliable main effect for the factor of drug groups $[F(4,25)=3.93, p<$ .02] and a reliable interaction between drug group and trials $[\mathrm{F}(12,75)=5.48, \mathrm{p}<.001]$. The main effect for the factor of trials was not a reliable source of variance $[F(3,75)=1.30, p>.25]$.

One-way ANOVAs on individual trials indicate that there were no significant differences among
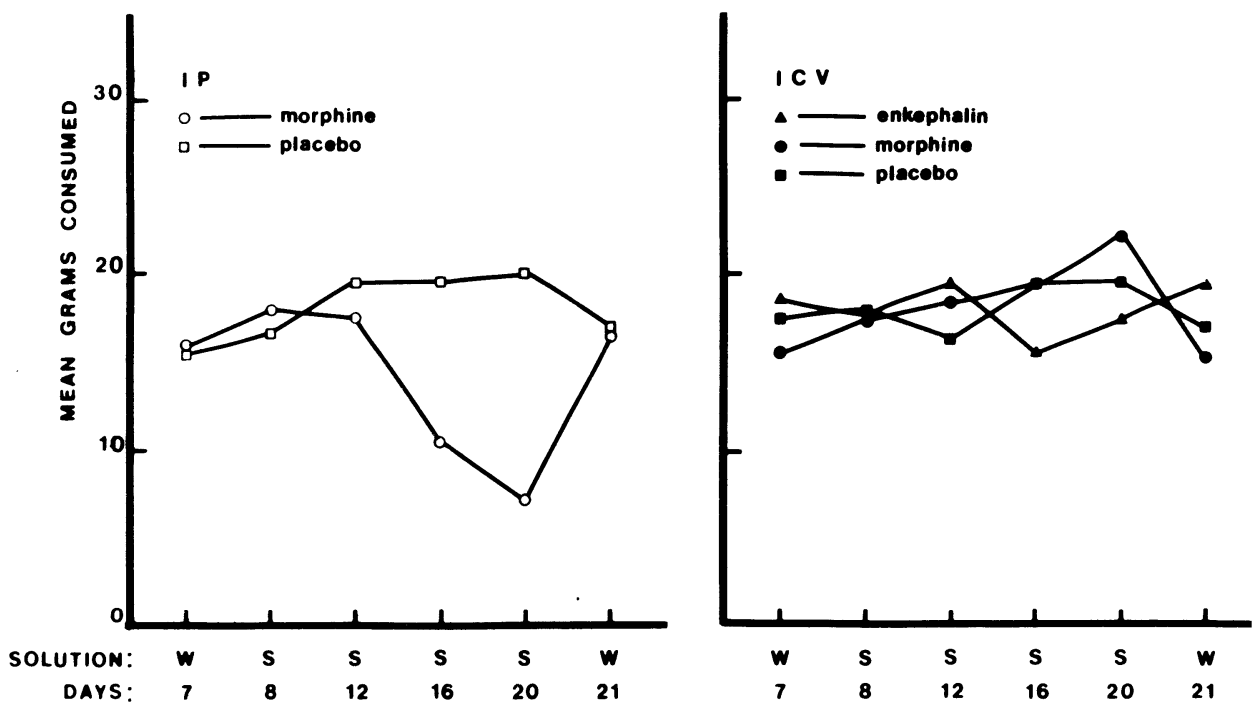

Figure 3. Mean amount of fluid consumed during a 15-min session on given days of the procedure. $\mathbf{W}=$ water as the solution presented, $\mathbf{S}=$ saccharin solution. Each group had a different drug administration following presentations of saccharin solution. 
groups on the first and second saccharin trials (Fs $<1$ ), but there were reliable differences on the third and fourth trials $[\mathrm{Fs}(4,25)=6.66,18.02$, respectively, ps $<.001$ ]. The systemic morphine group showed signs of developing a conditioned aversion to the saccharin solution, drinking significantly less on Trials 3 and 4 than placebo animals $[\operatorname{ts}(12)=4.47$, 8.33 , respectively, ps $<.001$ ] and less than their own preconditioning consumption on Trial 1 [ts(6) = $5.03,8.58$, respectively, ps $<.003$ ]. The enkephalin and ICV morphine groups showed no statistically significant differences from the ICV placebo group or their own preconditioning consumption.

Since the $10-\mathrm{mg} / \mathrm{kg}$ IP dose of morphine did produce a taste aversion, it can be inferred that this test was sensitive to such effects. Under these testing parameters, a $10-\mu \mathrm{g} \mathrm{ICV}$ dose of d-ala ${ }^{2}$-methionine enkephalin did not produce a poison aversion. This finding is concordant with the results of Switzman, Hammer, Shizgal, and Amit (1977). They found that doses as large as $148 \mu \mathrm{g}$ of $\mathrm{d}$-ala ${ }^{2}$-enkephalinamide were not sufficient to produce a taste aversion.

\section{GENERAL DISCUSSION}

There is an endorphin hypothesis of affective disorders, although currently support for such a hypothesis is weak (references in the introduction). Fundamental to such a hypothesis is evidence that endorphins can modify affect. This study does lead to the suggestion that increments in endorphins can result in a positive affective state, with few negative concomitants, and consequently provides some support for hypotheses linking endorphins to affect.

There are wide individual differences among rats in their readiness to consume sweetened morphine solutions (Gorman et al., 1978). These individual differences are related to a number of variables, but little is known about what might be a critical or fundamental variable. The finding that a history of high levels of enkephalin potentiates consumption leads to the suggestion that differences with respect to endorphins might be a fundamental difference among subjects that is functionally related to differences in readiness to consume morphine and to become addicted.

Lorens and Sainati (1978) have data showing that naloxone will attenuate addictive agents' potentiation of intracranial self-stimulation. From that data base, it has been suggested the endorphin system is involved with the positive affect elicited by addictive agents including opioids and ethanol. Critical to the confirmation of the suggestion is some evidence that activation of an endorphin system can elicit positive affect. Although alternative explanations are possible, the data of Experiments 1 and 3 do lead to the suggestion that activation of an endorphin system may lead to a positive affective state without aversive components. This observation is not incompatible with conclusions drawn by Belluzzi and Stein (1977). The results of Experiment 2 lead to the suggestion that activation of an endorphin system will potentiate one type of addictive behavior, namely propensity to take morphine solutions.

\section{REFERENCES}

Adams, W. J., Lorens, S. A., \& Mitchell, C. L. Morphine enhances lateral hypothalamic self-stimulation in the rat. Proceedings of the Society for Experimental Biology and Medicine, 1972, 140, 770-771.

Belluzzi, J. D., \& Stein, L. Enkephalin may mediate euphoria and drive-reduction reward. Nature, 1977, 266, 556-558.

Bloom, F., Segal, D., Ling, N., \& Guillemin, R. Endorphins: Profound behavioral effects in rats suggest new etiological factors in mental illness. Science, 1976, 194, 630-632.

Criswell, H. E., \& LevitT, R. A. The narcotic analgesics. In R. A. Levitt (Ed.), Psychopharmacology: A biological approach. Washington, D.C: Hemisphere, 1975.

Esposito, R. U., \& KoRNETSKy, C. Opioids and rewarding brain stimulation. Neuroscience and Biobehavioral Reviews, 1978, 2, 115-122.

Garcia, J., Hankins, W. G., \& Rusiniak, K. W. Behavioral regulation of the milieu interne in man and rat. Science, 1974, $185,824-831$.

Gorman, J. E., DeObaldia, R. N., Scott, R. C., \& REid, L. D. Morphine injections in the taste aversion paradigm: Extent of aversions and readiness to consume sweetened morphine solutions. Physiological Psychology, 1978, 6, 101-109.

Grevert, P., \& Goldstein, A. Endorphins: Naloxone fails to alter experimental pain or mood in humans. Science, 1978, 199, 1093-1095.

JaCQUET, Y. F., \& Marks, N. The C-fragment of $\beta$-lipotropin: An endogenous neuroleptic or antipsychotogen? Science, 1976, 194, 632-635.

Khavari, K. A., \& Risner, M. E. Opiate dependence produced by ad libitum drinking of morphine in water, saline, and sucrose vehicles. Psychopharmacologia, 1973, 30, 291-302.

LeBlanc, A. E., \& Cappell, H. Attenuation of punishing effects of morphine and amphetamine by chronic prior treatment. Journal of Comparative and Physiological Psychology, 1975, 87, 691-698.

Lorens, S. A., \& Sainati, S. M. Opiate receptors mediate the excitatory (euphorigenic) effect of ethanol, chlordiazepoxide and morphine on brain stimulation reward. Society for Neuroscience Abstracts, 1978, 4, 135. (Abstract)

McAuliffe, W. E., \& Gordon, R. A. A test of Lindesmith's theory of addiction: The frequency of euphoria among longterm addicts. American Journal of Sociology, 1974, 79, 795-840.

Parker, L., Failor, A., \& Weidman, K. Conditioned preferences in the rat with an unnatural need state: Morphine withdrawal. Journal of Comparative and Physiological Psychology, 1973, 82, 294-300.

Reid, L. D., Lind, M. D., Bozarth, M. A., Merriman, V. J., \& Stapleton, J. M. Small doses of morphine sulfate and pressing for hypothalamic intracranial stimulation (ICS) in rats. Society for Neuroscience Abstracts, 1978, 4, 501. (Abstract)

RILEY, A. L., \& BARIL, L. L. Conditioned taste aversion: A bibliography. Animal Learning \& Behavior, 1976, 4, 1S-13S. (Suppl.)

Riley, A. L., Jacobs, W. J., \& Lolordo, V. M. Morphine induced taste aversions: A consideration of parameters. Physiological Psychology, 1978, 6, 96-100.

Rossi, N. A., \& REID, L. D. Affective states associated with 
morphine injections. Physiological Psychology, 1976, 4, 269-274.

Switzman, L., Hammer, L., Shizgal, P., \& Amit, Z. A paradoxical "aversive" property of leucine-enkephalin: Conditioned taste aversion in rats. Society for Neuroscience Abstracts, 1977, 3, 303. (Abstract)

Van der Kooy, D., Le Piane, F. G., \& Phillips, A. G. Apparent independence of opiate reinforcement and electrical selfstimulation systems in rat brain. Life Sciences, 1977, 20, 981-986.

Walker, J. M., Sandman, C. A., Berntson, G. G., McGivern, R. R., Coy, D. H., \& Kastin, A. J. Endorphin analogs with potent and long-lasting analgesic effect. Pharmacology, Biochemistry, \& Behavior, 1977, 7, 543-548.
Watson, S. J., Berger, P. A., Akil, H., Mills, M. J., \& BARCHAS, J. D. Effects of naloxone on schizophrenia: Reduction in hallucinations in a subpopulation of subjects. Science, 1978, 201, 73-75.

WIKLER, A. Requirements for extinction of relapse-facilitating variables and for rehabilitation in a narcotic-antagonist treatment program. In M. C. Braude, L. S. Harris, E. C. May, J. P. Smith, \& J. E. Villarreal (Eds.), Advances in biochemical psychopharmacology (Vol. 8): Narcotic antagonists. New York: Raven Press, 1974.

(Received for publication October 27, 1978; revision accepted February 23, 1979.) 